\title{
On the norm-closure of the class of hypercyclic operators
}

\author{
by Christoph Schmoeger (Karlsruhe)
}

\begin{abstract}
Let $T$ be a bounded linear operator acting on a complex, separable, infinitedimensional Hilbert space and let $f: D \rightarrow \mathbb{C}$ be an analytic function defined on an open set $D \subseteq \mathbb{C}$ which contains the spectrum of $T$. If $T$ is the limit of hypercyclic operators and if $f$ is nonconstant on every connected component of $D$, then $f(T)$ is the limit of hypercyclic operators if and only if $f\left(\sigma_{\mathrm{W}}(T)\right) \cup\{z \in \mathbb{C}:|z|=1\}$ is connected, where $\sigma_{\mathrm{W}}(T)$ denotes the Weyl spectrum of $T$.
\end{abstract}

1. Terminology and introduction. In this note $X$ always denotes a complex, infinite-dimensional Banach space and $\mathcal{L}(X)$ the Banach algebra of all bounded linear operators on $X$. We write $\mathcal{K}(X)$ for the ideal of all compact operators on $X$. For $T \in \mathcal{L}(X)$ the spectrum of $T$ is denoted by $\sigma(T)$. The reader is referred to [5] for the definitions and properties of Fredholm operators, semi-Fredholm operators and the index ind $(T)$ of a semi-Fredholm operator $T$ in $\mathcal{L}(X)$. For $T \in \mathcal{L}(X)$ we will use the following notations:

$$
\begin{aligned}
\varrho_{F}(T)= & \{\lambda \in \mathbb{C}: \lambda I-T \text { is Fredholm }\}, \\
\varrho_{\mathrm{s}-\mathrm{F}}(T)= & \{\lambda \in \mathbb{C}: \lambda I-T \text { is semi-Fredholm }\}, \\
\varrho_{\mathrm{W}}(T)= & \left\{\lambda \in \varrho_{\mathrm{F}}(T): \text { ind }(\lambda I-T)=0\right\}, \\
\sigma_{0}(T)= & \left\{\lambda \in \sigma(T): \lambda \text { is isolated in } \sigma(T), \text { and } \lambda \in \varrho_{\mathrm{F}}(T)\right\}, \\
\sigma_{\mathrm{F}}(T)= & \mathbb{C} \backslash \varrho_{\mathrm{F}}(T), \quad \sigma_{\mathrm{s}-\mathrm{F}}(T)=\mathbb{C} \backslash \varrho_{\mathrm{s}-\mathrm{F}}(T), \\
\sigma_{\mathrm{W}}(T)= & \mathbb{C} \backslash \varrho_{\mathrm{W}}(T) \quad(\text { Weyl spectrum }), \\
\operatorname{Hol}(T)= & \{f: D(f) \rightarrow \mathbb{C}: D(f) \text { is open, } \sigma(T) \subseteq D(f), \\
& f \text { is holomorphic }\}, \\
\widetilde{\operatorname{Hol}(T)=} & \{f \in \operatorname{Hol}(T): f \text { is nonconstant on every connected } \\
& \text { component of } D(f)\} .
\end{aligned}
$$

1991 Mathematics Subject Classification: Primary 47B99.

Key words and phrases: hypercyclic operators. 
For $f \in \operatorname{Hol}(T)$, the operator $f(T)$ is defined by the well known analytic calculus (see [5]).

If $X$ is separable, then $T \in \mathcal{L}(X)$ is called hypercyclic if $\left\{x, T x, T^{2} x, \ldots\right\}$ is dense in $X$ for some $x \in X$. We denote by $\mathcal{H C}(X)$ the class of all hypercyclic operators in $\mathcal{L}(X)$. The following simple spectral description of the norm-closure $\mathcal{H C}(X)^{-}$is due to D. A. Herrero [3], Theorem 2.1:

TheOrem 1. If $X$ is a separable Hilbert space, then $A \in \mathcal{H C}(X)^{-}$if and only if $A$ satisfies the conditions

(1) $\sigma_{\mathrm{W}}(A) \cup\{z \in \mathbb{C}:|z|=1\}$ is connected,

(2) $\sigma_{0}(A)=\emptyset$, and

(3) $\operatorname{ind}(\lambda I-A) \geq 0$ for all $\lambda \in \varrho_{\mathrm{s}-\mathrm{F}}(A)$.

Furthermore, $\mathcal{H C}(X)^{-}+\mathcal{K}(X)=\{A \in \mathcal{L}(X): A$ satisfies (1) and (3) $\}$.

The main result of the present note reads as follows:

Theorem 2. Let $X$ be a separable Hilbert space, $T \in \mathcal{H C}(X)^{-}$and let $f \in \widehat{\operatorname{Hol}(T)}$. Then the following assertions are equivalent:

(1) $f(T) \in \mathcal{H C}(X)^{-}$.

(2) $f(T) \in \mathcal{H C}(X)^{-}+\mathcal{K}(X)$.

(3) $f\left(\sigma_{\mathrm{W}}(T)\right) \cup\{z \in \mathbb{C}:|z|=1\}$ is connected.

As an immediate consequence we have:

Corollary. Let $X, T$ and $f$ be as in Theorem 2. If $\sigma_{\mathrm{W}}(T)$ is connected and $\left|f\left(\lambda_{0}\right)\right|=1$ for some $\lambda_{0} \in \sigma_{\mathrm{W}}(T)$, then $f(T) \in \mathcal{H C}(X)^{-}$.

A result closely related to the above corollary can be found in [4], Theorem 2 .

The proof of Theorem 2 will be given in Section 3 of this paper. For this proof we need some preliminary results, which we collect in Section 2. Many of these preliminary results can be found in [1], Section 3, in the Hilbert space case.

2. Preliminary results. In this section $X$ will denote an arbitrary complex Banach space.

Proposition 1. Let $T \in \mathcal{L}(X)$ and $f \in \operatorname{Hol}(T)$.

(1) $f\left(\sigma_{\mathrm{F}}(T)\right)=\sigma_{\mathrm{F}}(f(T))$.

(2) $f\left(\sigma_{\mathrm{s}-\mathrm{F}}(T)\right) \subseteq \sigma_{\mathrm{s}-\mathrm{F}}(f(T))$ (if $f$ is univalent, we have equality).

(3) If $f \in \widehat{\operatorname{Hol}(T)}$, then $\sigma_{0}(f(T)) \subseteq f\left(\sigma_{0}(T)\right)$.

(4) If $\operatorname{ind}(\lambda I-T) \geq 0$ for all $\lambda \in \varrho_{\mathrm{F}}(T)$ or $\operatorname{ind}(\lambda I-T) \leq 0$ for all $\lambda \in \varrho_{\mathrm{F}}(T)$, then

$$
\sigma_{\mathrm{W}}(f(T))=f\left(\sigma_{\mathrm{W}}(T)\right) .
$$


Proof. (1) $\sigma_{\mathrm{F}}(T)$ is the spectrum of $T+\mathcal{K}(X)$ in the Banach algebra $\mathcal{L}(X) / \mathcal{K}(X)$. Hence the spectral mapping theorem holds for $\sigma_{\mathrm{F}}(T)$.

(2) See [6], Corollary 1, or [2], Theorem 1.

(3) Let $\mu_{0} \in \sigma_{0}(f(T))$; thus $\mu_{0}$ is an isolated point in $\sigma(f(T))=f(\sigma(T))$ and $\mu_{0} \in \varrho_{\mathrm{F}}(f(T))$. We have $\mu_{0}=f\left(\lambda_{0}\right)$ for some $\lambda_{0} \in \sigma(T)$. By $(1), \lambda_{0} \in$ $\varrho_{\mathrm{F}}(T)$. Let $C$ denote the connected component of $D(f)$ which contains $\lambda_{0}$. Assume that $\lambda_{0}$ is not isolated in $\sigma(T)$, thus there is a sequence $\left(\lambda_{n}\right)$ in $C \cap \sigma(T)$ such that $\lambda_{n} \rightarrow \lambda_{0}$ and $\lambda_{n} \neq \lambda_{0}$ for all $n \in \mathbb{N}$. This gives $f\left(\lambda_{n}\right) \rightarrow$ $f\left(\lambda_{0}\right)=\mu_{0}(n \rightarrow \infty)$. Since $f\left(\lambda_{n}\right) \in f(\sigma(T))=\sigma(f(T))$ and $\mu_{0}$ is isolated in $\sigma(f(T))$, we derive $f\left(\lambda_{n}\right)=\mu_{0}$ for all $n$. By the uniqueness theorem for analytic functions, it follows that $f(\lambda)=\mu_{0}$ for all $\lambda \in C$, a contradiction. Thus $\lambda_{0}$ is an isolated point in $\sigma(T)$. Since $\lambda_{0} \in \varrho_{\mathrm{F}}(T)$, we get $\lambda_{0} \in \sigma_{0}(T)$, hence $\mu_{0}=f\left(\lambda_{0}\right) \in f\left(\sigma_{0}(T)\right)$.

(4) follows from [8], Theorem 3.6.

R e mark. In general, the spectral mapping theorem for the Weyl spectrum $\sigma_{\mathrm{W}}(T)$ does not hold (see [2], p. 23, or [8], Example 3.3).

Notations. For $T \in \mathcal{L}(X)$, we write $\alpha(T)$ for the dimension of the kernel of $T$ and $\beta(T)$ for the co-dimension of the range of $T$. Thus, if $T$ is semi-Fredholm,

$$
\operatorname{ind}(T)=\alpha(T)-\beta(T) \in \mathbb{Z} \cup\{-\infty,+\infty\} .
$$

According to C. Pearcy [7], the next proposition has already appeared in the preprint Fredholm operators by P. R. Halmos in 1967. For the convenience of the reader we shall include a proof.

Proposition 2. If $T$ and $S$ are semi-Fredholm operators with $\alpha(T)$ and $\alpha(S)$ finite $[$ resp. $\beta(T)$ and $\beta(S)$ finite $]$, then TS is a semi-Fredholm operator with $\alpha(T S)<\infty[$ resp. $\beta(T S)<\infty]$ and

$$
\operatorname{ind}(T S)=\operatorname{ind}(T)+\operatorname{ind}(S) .
$$

Pr o of. It suffices to consider the case where $\alpha(T), \alpha(S)<\infty$.

Case 1: $T$ and $S$ are Fredholm operators. Then it is well known that $T S$ is Fredholm and ind $(T S)=\operatorname{ind}(T)+\operatorname{ind}(S)$ (see [5], §71).

Case 2: $T$ or $S$ is not Fredholm. Thus $\beta(T)=\infty$ or $\beta(S)=\infty$. Use [5], $\S 82$, Aufgaben 2, 4, to get: TS is semi-Fredholm, $\alpha(T S)<\infty, \beta(T S)=\infty$. Hence ind $(T S)=-\infty=\operatorname{ind}(T)+\operatorname{ind}(S)$.

Proposition 3. Let $T \in \mathcal{L}(X)$ satisfy

$$
\sigma_{0}(T)=\emptyset \quad \text { and } \quad \operatorname{ind}(\lambda I-T) \geq 0 \quad \text { for all } \lambda \in \varrho_{\mathrm{s}-\mathrm{F}}(T) .
$$

If $f \in \widetilde{\operatorname{Hol}(T)}$ then we have 
(1) $\sigma_{0}(f(T))=\emptyset$

(2) $\operatorname{ind}(\mu I-f(T)) \geq 0$ for all $\mu \in \varrho_{\mathrm{s}-\mathrm{F}}(f(T))$.

Proof. (1) follows from Proposition 1(3).

(2) Take $\mu_{0} \in \varrho_{\mathrm{s}-\mathrm{F}}(f(T))$ and put $g(\lambda)=\mu_{0}-f(\lambda)$. If $g$ has no zeroes in $\sigma(T)$, then $g(T)=\mu_{0} I-f(T)$ is invertible in $\mathcal{L}(X)$, thus ind $\left(\mu_{0} I-f(T)\right)$ $=0$. If $g$ has zeroes in $\sigma(T)$, then $g$ has only a finite number of zeroes in $\sigma(T)$, since $f \in \widetilde{\operatorname{Hol}(T)}$. Let $\lambda_{1}, \ldots, \lambda_{k}$ be those zeroes and $\nu_{1}, \ldots, \nu_{k}$ their respective orders. Then we have

$$
g(\lambda)=h(\lambda) \prod_{j=1}^{k}\left(\lambda_{j}-\lambda\right)^{\nu_{j}}
$$

with $h \in \operatorname{Hol}(T)$ and $h(\lambda) \neq 0$ for all $\lambda \in \sigma(T)$. Therefore $h(T)$ is invertible and

$$
g(T)=h(T) \prod_{j=1}^{k}\left(\lambda_{j} I-T\right)^{\nu_{j}} .
$$

Since $0 \in \varrho_{\mathrm{s}-\mathrm{F}}(g(T))$, we get, by Proposition $1(2)$,

$$
\lambda_{1}, \ldots, \lambda_{k} \in \varrho_{\mathrm{s}-\mathrm{F}}(T) .
$$

Since ind $\left(\lambda_{j} I-T\right) \geq 0$, we have

$$
\beta\left(\lambda_{j} I-T\right)<\infty \quad \text { for } j=1, \ldots, k .
$$

Thus by Proposition 2 (recall that $\beta(h(T))=0<\infty$ ),

$$
\begin{aligned}
\operatorname{ind}\left(\mu_{0} I-f(T)\right) & =\operatorname{ind}(g(T)) \\
& =\underbrace{\operatorname{ind}(h(T))}_{=0}+\sum_{j=1}^{k} \nu_{j} \underbrace{\operatorname{ind}\left(\lambda_{j} I-T\right)}_{\geq 0} \geq 0 .
\end{aligned}
$$

R e m a r k. The description of the index in [1], Theorem 3.7, sheds more light on claim (4) of Proposition 1 and on claim (2) of Proposition 3 in the Hilbert space case.

3. Proof of Theorem 2. $(1) \Rightarrow(2)$. Clear.

$(3) \Rightarrow(1)$. Use Proposition 1(4), Proposition 3 and Theorem 1.

$(2) \Rightarrow(3)$. By Theorem $1, \sigma_{\mathrm{W}}(f(T)) \cup\{z \in \mathbb{C}:|z|=1\}$ is connected. Use again Proposition 1(4) to derive (3). 


\section{References}

[1] C. Bosch, C. Hernández, E. De Oteyza and C. Pearcy, Spectral pictures of functions of operators, J. Operator Theory 8 (1982), 391-400.

[2] B. Gramsch and D. Lay, Spectral mapping theorems for essential spectra, Math. Ann. 192 (1971), 17-32.

[3] D. A. Herrero, Limits of hypercyclic and supercyclic operators, J. Funct. Anal. 99 (1991), 179-190.

[4] G. Herzog and C. Schmoeger, On operators $T$ such that $f(T)$ is hypercyclic, Studia Math. 108 (1994), 209-216.

[5] H. Heuser, Funktionalanalysis, 2nd ed., Teubner, Stuttgart, 1986.

[6] K. K. Oberai, Spectral mapping theorem for essential spectra, Rev. Roumaine Math. Pures Appl. 25 (1980), 365-373.

[7] C. Pearcy, Some Recent Developments in Operator Theory, CBMS Regional Conf. Ser. in Math. 36, Amer. Math. Soc., Providence, 1978.

[8] C. Schmoeger, Ascent, descent and the Atkinson region in Banach algebras, II, Ricerche Mat. 42 (1993), 249-264.

Mathematisches Institut I

Universität Karlsruhe

D-76128 Karlsruhe, Germany

Reçu par la Rédaction le 21.8.1995

Révisé le 10.4.1996 